\title{
A Systematic Review on Practical Considerations, Recent Advances and Research Challenges in Underwater Optical Wireless Communication
}

\author{
Syed Agha Hassnain Mohsan ${ }^{1}$ Md. Mehedi Hasan ${ }^{2}$, Alireza Mazinani ${ }^{3}$ \\ Muhammad Abubakar Sadiq ${ }^{4}$, Muhammad Hammad Akhtar ${ }^{5}$, Asad Islam ${ }^{6}$, Laraba Selsabil Rokia ${ }^{7}$ \\ Department of Electrical Engineering, COMSATS University Islamabad (CUI), Islamabad, Pakistan ${ }^{1}$ \\ Department of Electronics and Information Engineering, Beihang University, Beijing, China ${ }^{2}$ \\ School of Electronic and Information Engineering, Beihang University, Beijing, China ${ }^{3}$ \\ Department of Computer Science and Engineering, Beihang University (BUAA), Beijing, China ${ }^{4}$ \\ Research Centre for Modeling and Simulation, National University of Science and Technology (NUST), Islamabad, Pakistan ${ }^{5}$ \\ School of Energy and Power Engineering, Beihang University (BUAA), Beijing, China ${ }^{6}$ \\ Department of Biomaterials, East China University of Science and Technology, Shanghai, China ${ }^{7}$
}

\begin{abstract}
Underwater Optical Wireless Communication (UOWC) has gained significant attraction in many underwater activities because of its high bandwidth as compared to radio frequency and acoustic technologies. Underwater Optical Wireless Communication (UOWC) has high stature in underwater observation, exploration and monitoring applications. However, due to complex nature of ocean water, several practical challenges exist in deployment of UOWC links. Qualitative and effective research has been carried out in UOWCs from last few decades. Ambition behind this research systematic study is to provide a comprehensive survey on latest research in UOWCs. Herein, we provide a brief discussion on major research challenges, limitations and development in UOWCs. We provide a periodical review on UOWC issues and potential challenges highlighted in previous studies. In this paper, we have also investigated research methods to gain attention of research fraternity towards future technologies and challenges on the basis of existing approaches. Thus, it is our foremost requirement to provide state-of-the-art analysis of existing UOWCs. Significant deliberation has been provided with recent bibliography.
\end{abstract}

Keywords-Component; underwater optical wireless communication; underwater technologies; research questions; $5 G / 6 G$

\section{INTRODUCTION}

An extensive research has been carried out on free space optical wireless communications in different aspects and nowadays Underwater Optical Wireless Communication (UOWC) is generating considerable attention among recent researchers. UOWC is an emerging technology for premier research in underwater environment. It is a fusion of optical and wireless technologies having intelligent computing, smart sensing and communication abilities. UOWC is used in numerous applications such as mineral exploration, ocean observation, submarine communication, diver-to-diver communication, military applications, underwater navigation and surveillance. UOWC has successfully proven its stature in target detection, objection tracking, underwater robotics, monitoring, AUVs communication and oceanographic data transmission and collection. Though UOWC is providing cutting-edge solutions but many challenges exist at the same time. The researchers face challenges regarding link deployment, propagation delay, high bit error rates, Doppler spread, connectivity losses, attenuation, scattering, turbulence, low bandwidth, high latency, multipath propagation and salinity. The unpredictable condition of ocean environment generates these serious challenges and issues in designing and deployment of UOWC links. In this paper, we have investigated several challenges and presented our work summary on it. We have discussed several constraints and particularities in this systematic review. We have briefly discussed the feasible countermeasures to tackle these challenges in existing UOWC mechanism. UOWCs are vulnerable to numerous factors such as scattering, absorption and fading. In addition, UOWC performance is limited to short range [1] and extensive research is required for long range communications. Researchers are working to design systems and methodologies to transmit broadband optical signals at larger distances. In near future, submarine optical communication systems will be available commercially [2]. A general UOWC system is displayed in Fig. 1. It shows that different underwater platforms such as submarine, sensors, ships and divers are connected through light beams.

An extensive research is being carried out to develop a complementary technology which can allow broadband underwater communications such as real-time video transmissions, tele-operation of AUVs and remote monitoring of underwater stations [4]. In future, optical communication will be used for many underwater applications. However, UOWC cannot fully replace acoustic communications. For this purpose, scientists are focusing on hybrid optic/acoustic communications [5].

We have briefly discussed several potential features in our systematic review. We have included modulation schemes, channel coding, UOWC challenges and OWC revolution in 
5G and 6G networks. We have organized this paper as shown in Fig. 2.

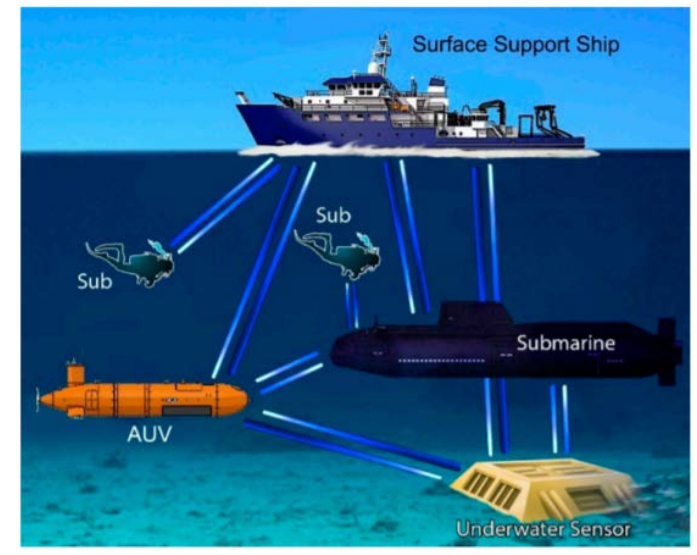

Fig. 1. UOWC System [3].

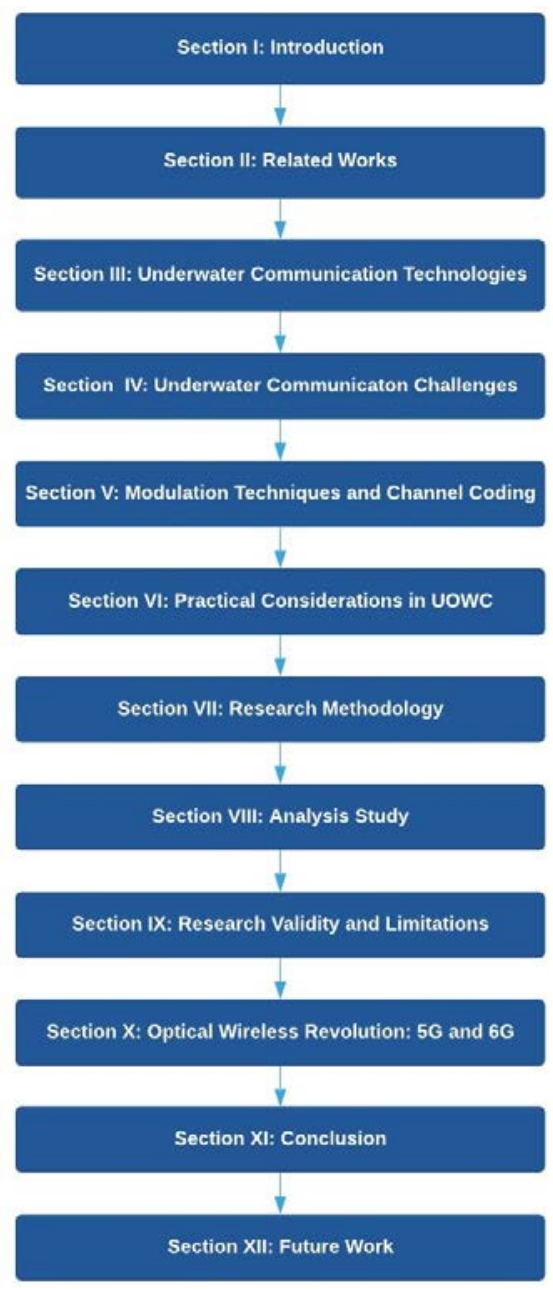

Fig. 2. Overview of Reseach Work.

\section{RELATED WORK}

In this section, we have summarized practical considerations and issues in UOWCs in existing research literature. We provide an overview of UOWC technology, enhancing functions and issues highlighted in recent research publications. Previous researchers have provided scholarly discussions about communication technologies, technical aspects and challenges of UOWC. In a recent research study conducted in 2020 [6], researchers have provided a review on practical consideration and solution in UWOCs. They have addressed underwater challenges and possible solutions. They have discussed misalignment in underwater links and highlighted novel solutions to bring robustness in UWOC links. An exhaustive and inclusive survey of state-of-the-art UOWC is given in which authors have considered aspects of coding techniques, modulation and channel characterization [7] in UOWCs. A good scholarly approach based on overview of current advances in UOWC is given in an article [8]. In this study, authors have focused the reliability and feasibility of optical links with higher data rates. Emerging optical wireless communications, transcutaneous OWCs, optical scattering and free space optics are discussed in a previous study [9]. Khalighi et al. [10] have given a performance study of typical UOWC system and addressed open issues. Performance of FSO systems and various impairments which degrade FSO performance are highlighted in [11]. Authors have discussed FSO modulation, coding schemes using various detection techniques. In a Master's thesis, Z. Zeng has given an interesting survey about optical wireless communications [12]. Author has provided good remarks for UWOC channel modeling, channel modulation and coding techniques. Weihao Liu et al. provided a roadmap to characterize an UOWC channel with considering both underwater optical turbulence and absorption/scattering [13]. Pal A. van Walree carefully addressed scattering, propagation and fading effects [14]. Researchers have conducted a brief survey on recent advances, research activities and potential applications [15] of OWC. Aobo Lin et al. [16] successfully demonstrated UOWC by carrying out experiments with blue LEDs. A blue GaNbased micro-LED was used to achieve high speed in UOWC [17]. An efficient diver-to-diver communication system was demonstrated in [18], researchers claimed to transmit audio signal by using LEDs of different wavelengths. Peppas et al. [19] have discussed performance of UOWC systems and investigated effects of oceanic turbulence and inter-symbol interference. Rashed et al. [20] have constructed an underwater link budget for realistic ocean water by considering scattering and absorption. Depth dependent variation in attenuation and beam refraction are addressed by LJ Johnson [21-22]. He also outlined transmission characteristics for UOWC in [23]. A concise review on UOWCs and hidden abilities of optical spectrum is addressed [24]. Authors in [25] performed experimental trials to check optimum LED wavelengths for UOWCs. Opportunities and challenges in OWC technologies are highlighted in a study [26]. The effects of collimated laser beams in UOWC links are investigated [27]. D. Anguita et al. have proposed a UOWC model for AUVs [28]. Optical amplification and spatial diversity for UOWC is discussed in a previous study [29]. Johnson et al. have focused on the impact of link orientation in UOWC [30]. UOWC system for real time swimmers is designed by some researchers [31]. They have also addressed air bubbles effect in their study. Table I summarizes the research work and key aspects in different years. 
TABLE I. RESEARCH WORK

\begin{tabular}{|l|l|l|}
\hline Reference \# & Focused Key Areas & Year \\
\hline$[32]$ & OWC for UWSNs & 2010 \\
\hline$[28]$ & UOWC applications & 2011 \\
\hline$[31]$ & Real time application of UOWC & 2012 \\
\hline$[20]$ & Underwater link budget & 2013 \\
\hline$[21]$ & Attenuation in water & 2013 \\
\hline$[10]$ & UOWC recent dancesandcllnges & 2014 \\
\hline$[22]$ & Beam refraction in water & 2014 \\
\hline$[23]$ & Transmission characterictis in UOWC & 2014 \\
\hline$[30]$ & Link orientation in UOWC & 2014 \\
\hline$[9]$ & OWC advances and challenges & 2015 \\
\hline$[13]$ & $\begin{array}{l}\text { Turbulence effect and detection } \\
\text { schemes for UWOC }\end{array}$ & 2015 \\
\hline$[15]$ & $\begin{array}{l}\text { Recent advances and potential } \\
\text { applications }\end{array}$ & 2015 \\
\hline$[7]$ & Link misalignment issues & 2016 \\
\hline$[29]$ & $\begin{array}{l}\text { Optical amplification and spatial } \\
\text { diversity }\end{array}$ & 2016 \\
\hline$[19]$ & Oceanic turbulence effect and ISI & 2017 \\
\hline$[26]$ & Opportunities and challenges & 2017 \\
\hline$[33]$ & Turbulence effect on UOWC & 2018 \\
\hline$[13]$ & $\begin{array}{l}\text { Research challenges and roadmaps for } \\
\text { UWOC }\end{array}$ & 2019 \\
\hline$[6]$ & $\begin{array}{l}\text { Practical considerations and solution } \\
\text { for UOWC }\end{array}$ & 2020 \\
\hline$[3]$ & Comparative analysis of UWOC & 2020 \\
\hline & & \\
\hline
\end{tabular}

\section{UNDERWATER COMMUNICATION TECHNOLOGIES}

Current underwater optical systems consider acoustic waves as EM waves cannot travel properly. However, acoustic waves have limitation in aspects of limited bandwidth and slow speed. Acoustic communication is preferred for long distance but optical communication gives better performance for short distance. Light signals lose energy and change direction due to water particles. These challenging factors limit the range of communication system.

Table II presents characteristics comparison between existing underwater wireless technologies.

We have plotted a performance comparison of the four channels by considering their data rate and transmission range in MATLAB as shown in Fig. 3.

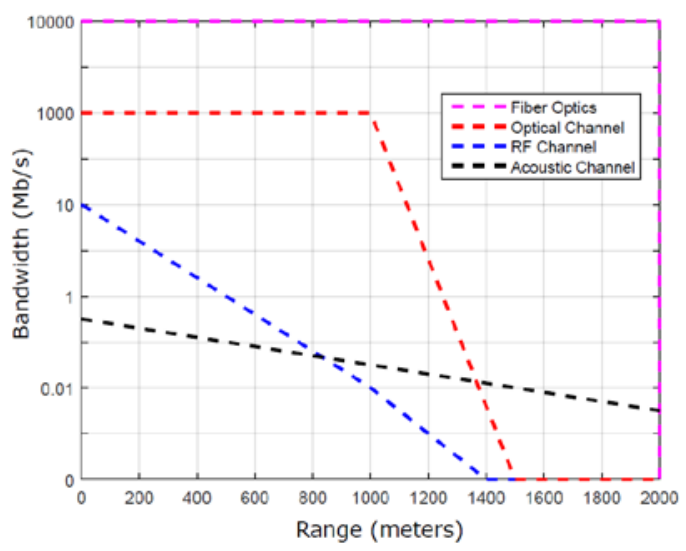

Fig. 3. Transmission Channels.
TABLE II. CHARACTERISTICS OF UNDERWATER TECHNOLOGIES

\begin{tabular}{|l|l|l|l|}
\hline Characteristics & Optical & Acoustic & Radio Frequency \\
\hline $\begin{array}{l}\text { Performance } \\
\text { Parameter }\end{array}$ & $\begin{array}{l}\text { Scattering, } \\
\text { Absorption }\end{array}$ & $\begin{array}{l}\text { Pressure, } \\
\text { Salinity and } \\
\text { Temperature }\end{array}$ & $\begin{array}{l}\text { Permitivitty, } \\
\text { Permeability and } \\
\text { Conductivity } \\
\text { [38,39] }\end{array}$ \\
\hline $\begin{array}{l}\text { Transmission } \\
\text { Power }\end{array}$ & Few Watts & Tens of Watts & $\begin{array}{l}\text { mW to hundreds of } \\
\text { Watts }\end{array}$ \\
\hline Bandwidth & $10-150 \mathrm{MHz}$ & $\begin{array}{l}1000 \mathrm{~km}< \\
1 \mathrm{kHz} \\
\text { and } \\
\mathrm{kHz} \\
\text { and } \\
<100 \mathrm{~mm} \approx 100 \\
\mathrm{kHz}\end{array}$ & MHz \\
\hline Latency & Low & High & Medium \\
\hline Attenuation & $\begin{array}{l}0.39 \mathrm{~dB} / \mathrm{m} \\
\text { Ocean }\end{array}$ & $0.1-4 \mathrm{~dB} / \mathrm{km}$ & $3.5-5 \mathrm{~dB} / \mathrm{m}$ \\
\hline Size of Antenna & $0.1 \mathrm{~m}$ & $0.1 \mathrm{~m}$ & - \\
\hline Data Rate & $\mathrm{Gbps}$ & $\mathrm{kbps}$ & $\mathrm{mbps}$ \\
\hline Efficiency & $\begin{array}{l}30 \\
\mathrm{kbits} / \mathrm{Joules}\end{array}$ & $100 \mathrm{bits} / \mathrm{Joules}$ & \\
\hline Speed & $2.2 \times 10^{8}$ & $1500 \mathrm{~m} / \mathrm{s}$ & $2.2 \times 10^{8}$ \\
\hline Frequency Band & $10^{12}-10^{15} \mathrm{~Hz}$ & $10-15 \mathrm{kHz}$ & $20-300 \mathrm{~Hz}(\mathrm{ELF})$ \\
\hline Distance & $10-100 \mathrm{~m}$ & $\mathrm{Up} \mathrm{to} \mathrm{kms}$ & Up to $10 \mathrm{~m}$ \\
\hline
\end{tabular}

Table III summarizes the benefits and limitations of different technologies implemented to achieve UWC.

TABLE III. COMPARISON OF UNDERWATER TECHNOLOGIES

\begin{tabular}{|c|c|c|}
\hline $\begin{array}{l}\text { UWC } \\
\text { Technologies }\end{array}$ & Limitations & Benefits \\
\hline Optical & $\begin{array}{ll}- & \begin{array}{l}\text { Severe scattering and } \\
\text { absorption }\end{array} \\
- & \text { Difficulty to cross } \\
\text { air/water interface } \\
\text { - } & \text { Medium range }\end{array}$ & $\begin{array}{ll}- & \text { Low cost } \\
- & \text { Lightweight } \\
- & \text { High data rate } \\
- & \text { Immune to } \\
& \text { latency }\end{array}$ \\
\hline $\begin{array}{l}\text { Radio } \\
\text { Frequency }\end{array}$ & $\begin{array}{ll}- & \text { Costly } \\
- & \text { Bulky } \\
- & \text { More energy consumption } \\
- & \text { Short range }\end{array}$ & $\begin{array}{ll}- & \text { Tolerant to } \\
\text { turbidity } \\
- & \text { Moderate data } \\
\text { rate } \\
-\quad \begin{array}{l}\text { Smoth flow from } \\
\text { air/water } \\
\text { interface }\end{array} \\
\end{array}$ \\
\hline Acoustic & $\begin{array}{ll}- & \text { High latency } \\
- & \text { More energy consumption } \\
- & \text { Costly } \\
- & \text { Dangerous for marine } \\
- & \text { species } \\
- & \text { Low data rate }\end{array}$ & $\begin{array}{ll}\text { - } & \text { Long distance } \\
\text { communication } \\
\text { - } \quad \text { Widely used } \\
\text { UWC } \\
\text { technology }\end{array}$ \\
\hline
\end{tabular}

\section{UNDERWATER COMMUNICATION CHALLENGES}

In this section, we have briefly discussed different potential challenges which degrade the performance of UOWC system.

\section{A. Scattering and Absorption Issues}

In optical region, waves are highly faded due to scattering and absorption. The extinction coefficient c of water medium is the attenuated beam over the complete path length. Extinction coefficient is the transmission wavelength which minimizes the propagation loss and it varies for different types 
of water. It is obtained by adding both absorption and scattering coefficients [34] a and b.

$c(\lambda)=a(\lambda)+b(\lambda)$

Table IV shows the color frequencies and respective wavelengths. Different color lights get absorbed at different water levels, where every water level has related spectrum characteristics.

Fig. 4 illustrates the absorption coefficient of pure seawater for different transmission wavelengths.

Extinction coefficient across a wide range of wavelengths in water is shown in Fig. 5. It can be concluded that blue light is useful for open ocean operations.

Table V provides the scattering, absorption, and extinction coefficient for different types of types of waters. Thus, we conclude demonstrating a UOCS closer to shore is more challenging than wide open ocean.

\section{B. Attenuation}

In water, less attenuation appears in wavelengths corresponding to blue green spectrum. However, optical link is associated to optical properties of the water and it varies according to geographic location. Attenuation $(\mathrm{dB} / \mathrm{m})$ variation against different wavelength ranges is shown in Fig. 6.

TABLE IV. LIGHT COLORS CHARACTERISTICS

\begin{tabular}{|l|l|l|}
\hline Colour & Frequency & Wavelength \\
\hline Red & $400-484 \mathrm{THz}$ & $620-750 \mathrm{~nm}$ \\
\hline Orange & $484-508 \mathrm{THz}$ & $590-620 \mathrm{~nm}$ \\
\hline Yellow & $508-526 \mathrm{THz}$ & $570-590 \mathrm{~nm}$ \\
\hline Blue & $631-668 \mathrm{THz}$ & $450-475 \mathrm{~nm}$ \\
\hline Green & $526-606 \mathrm{THz}$ & $495-570 \mathrm{~nm}$ \\
\hline
\end{tabular}

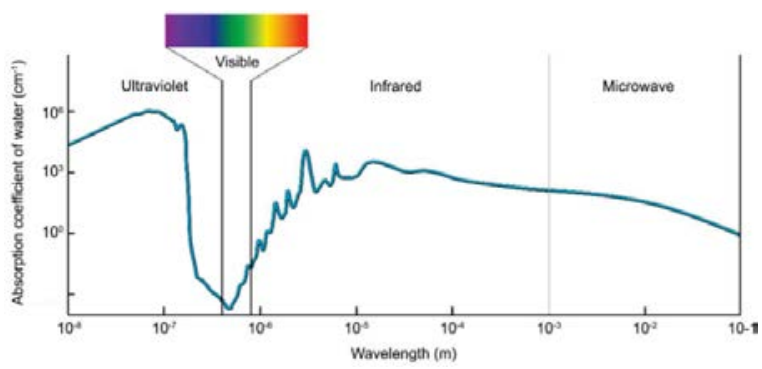

Fig. 4. Absorption Coefficient of Pure Water [3].

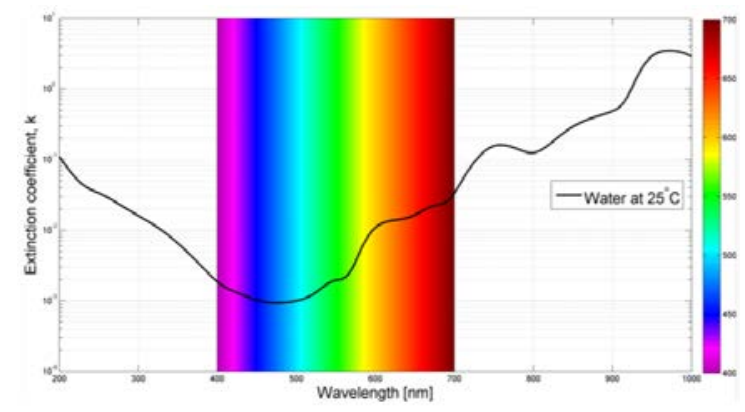

Fig. 5. Extinction Coefficient Variation [34].
TABLE V. OPTICAL PROPERTIES OF OCEAN WATER TyPes

\begin{tabular}{|l|l|l|l|l|}
\hline Water Type & $\mathbf{a ~ ( m - 1 )}$ & $\mathbf{b ~ ( m - 1 )}$ & $\mathbf{c ~ ( m - 1 )}$ & $\begin{array}{l}\mathbf{- 1 0 ~ d B} \\
\text { distance (m) }\end{array}$ \\
\hline Fresh Water & 0.040 & 0.02 & 0.042 & 53.55 \\
\hline Sea Water & 0.114 & 0.036 & 0.150 & 15.25 \\
\hline $\begin{array}{l}\text { Coastal } \\
\text { Water }\end{array}$ & 0.178 & 0.220 & 0.398 & 5.77 \\
\hline $\begin{array}{l}\text { Harbour } \\
\text { Water }\end{array}$ & 0.366 & 1.828 & 2.194 & 1.05 \\
\hline
\end{tabular}

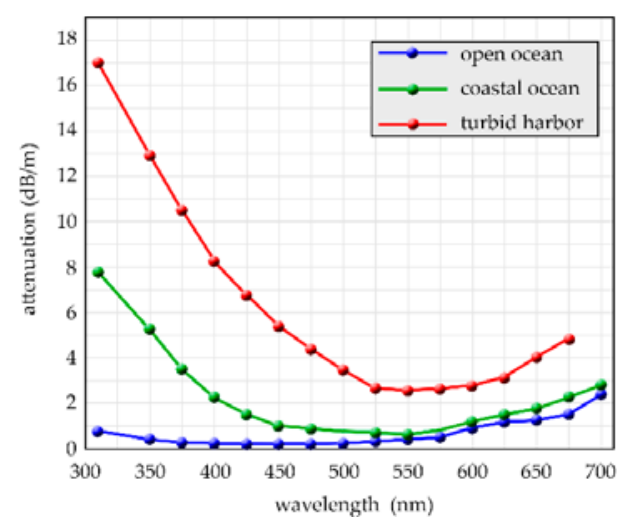

Fig. 6. Attenuation for different Waters [3].

\section{Underwater Link Alignment Issue}

Misalignment in optical transceivers can cause temporary disconnection in underwater optical links. Different UOWC systems make use of blue-green LEDs and lasers due to narrow divergence. However, it still requires a precise alignment [35]. A frequent misalignment is noticed at shallow depths; especially it occurs in applications based on vertical surface-to-bottom communication [21], [36]. Connection loss problem [37] occurs in case of random movements of sea surface.

\section{UOWC Devices}

Implementation and integration of UOWC systems needs appropriate and reliable underwater devices. Underwater characteristics such as pressure, salinity, flow and temperature highly influence the system performance and have strong impact on lifetime of UOWC devices. Energy efficient performance of whole UOWC system and reliability and sustainability of devices batteries is critical. Scholars should focus to design devices with low power consumption and higher lifetime.

\section{Modulation Techniques And Channel Coding}

Modulation schemes and coding techniques are discussed in this section. UOWC is referred as FSO communication in water, conventional FSO modulation techniques can be used in UOWC systems. PWM, OOK, DPIM and PPM are the most common intensity modulation techniques. OOK is the simplest scheme having low bandwidth efficiency. The selection of modulation schemes needs inclusive knowledge and in depth understanding application framework. OOK is preferred for discrete underwater sensor nodes as it is cost effective and simple. However, more than one modulation schemes are 
integrated to enhance the reliability and efficiency of UOWC system. Table VI gives performance comparison of different modulation schemes.

Different channel coding techniques are required to obtain low BER in UOWC system as it suffers from severe attenuation. Channel coding has benefits of low complexity, low implementation cost, error correction capability and low energy consumption. Thus, channel coding is appropriate for compact underwater sensors operating at high SNR in underwater environment. In order to enhance robustness, channel coding techniques such as Turbo and LDPC are integrated in main data processing node of UWSNs. We have summarized different coding techniques in Table VII.

TABLE VI. UOWC MODULATION SCHEMES

\begin{tabular}{|l|l|l|}
\hline $\begin{array}{l}\text { Modulation } \\
\text { Scheme }\end{array}$ & Limitation & Benefit \\
\hline OOK & Low energy efficient & Low cost, Simple \\
\hline SIM & $\begin{array}{l}\text { Complicated devices, Average } \\
\text { power efficiency }\end{array}$ & $\begin{array}{l}\text { Low cost, Icrease } \\
\text { system capacity }\end{array}$ \\
\hline QAM & $\begin{array}{l}\text { High cost, Complex } \\
\text { implementation }\end{array}$ & $\begin{array}{l}\text { Good response to } \\
\text { noise, High spectral } \\
\text { efficiency }\end{array}$ \\
\hline PPM & $\begin{array}{l}\text { Complex transceiver, Low } \\
\text { bandwidth }\end{array}$ & High power efficiency \\
\hline PoISK & $\begin{array}{l}\text { Low data rate, Short distance } \\
\text { range }\end{array}$ & $\begin{array}{l}\text { High tolerance to } \\
\text { turbulence }\end{array}$ \\
\hline PSK & $\begin{array}{l}\text { High cost, Complex } \\
\text { implementation }\end{array}$ & $\begin{array}{l}\text { High receiver } \\
\text { sensitivity }\end{array}$ \\
\hline DPIM & $\begin{array}{l}\text { Complicated demodulation } \\
\text { devices, Error spread in } \\
\text { demodulation }\end{array}$ & $\begin{array}{l}\text { High bandwidth } \\
\text { efficiency }\end{array}$ \\
\hline
\end{tabular}

TABLE VII. ChANNEL CODING TECHNIQUes

\begin{tabular}{|l|l|l|}
\hline $\begin{array}{l}\text { Channel } \\
\text { Coding }\end{array}$ & Limitation & Benefit \\
\hline Turbo & Complex encoder and decoder & High error correction \\
\hline LDPC & Complex encoder and decoder & High error correction \\
\hline CRC & $\begin{array}{l}\text { Low error correction in low SNR } \\
\text { condition }\end{array}$ & $\begin{array}{l}\text { Simple error detection } \\
\text { codes }\end{array}$ \\
\hline RS & $\begin{array}{l}\text { Low error correction in low SNR } \\
\text { condition }\end{array}$ & $\begin{array}{l}\text { Robust and simple } \\
\text { encoder and decoder }\end{array}$ \\
\hline I.T & $\begin{array}{l}\text { Complicated system } \\
\text { implementation }\end{array}$ & Minimize packet loss \\
\hline BCH & $\begin{array}{l}\text { Low error correction in low SNR } \\
\text { condition }\end{array}$ & $\begin{array}{l}\text { Robust and simple } \\
\text { encoder and decoder }\end{array}$ \\
\hline
\end{tabular}

\section{PRACTICAL CONSIDERATIONS IN UOWC}

In past few years, considerable research interest has been given to UOWC but anomalies exist due to several challenges. In this section we have provided some future directions in UOWCs.

\section{A. Channel Modeling}

Researchers have designed simulation tools and close-toreality models for horizontal links but vertical links still need attention from research community. Another practical consideration is to resolve turbulence effect in UOWC. Though turbulence is not considerable in deep waters but it must be taken into account in shallow waters. A lack in channel modeling techniques exist which should deal accurately with turbulence. The difficulty in designing such models lies in the fact that turbulence is closely related to water conditions and operating scenarios.

\section{B. Efficient Transmission Techniques}

Another essential issue to be explored is to design an opposite signaling scheme for aquatic channel. Up to now, several coding and modulation techniques are involved in UOWC systems. An improved reliability and link performance can be obtained by considering underwater channel particularities in designing of these transmission schemes. Energy efficient modulation schemes are needed for data transmission in high turbid water and powerful channel coding is required at different layers including physical and data link.

\section{Mitigating Link Misalignment}

A major issue in underwater environment is power consumption. One solution is to reduce the intensity loss by minimizing the beam divergence. In such scenario, energy efficient techniques and solutions are crucially important for $\mathrm{Tx} / \mathrm{Rx}$ beam alignment and localization. These issues can be mitigated through designing smart $\mathrm{Tx} / \mathrm{Rx}$ and adding additional components like collimating lens in UOWCs. There are some challenging issues to implement end-to-end communication links, researchers need to investigate routing protocols and spatial diversity techniques. A common UOWC system with point-to-point link needs strict tracking and pointing mechanism especially in mobility scenario. Electronic beam steering and segmented FOV can solve the issue of tracking and pointing for narrow optical beams.

\section{RESEARCH METHODOLOGY}

In this section, we discuss the subject's selection process of our findings and presented the framework and approaches we used to identify our results.

\section{A. Approaches Selection}

Our investigation starts with selecting the research articles providing a survey or review on challenges in UOWCs. In order to find initial list of target research articles, we used Google Scholar search. Google Scholar helped us to obtain relevant research articles, peer-reviewed publications, abstracts, preprints and research surveys and technical reports. Google Scholar gave us confidence to complete results based on articles collected from Google search, ResearchGate and academic publishers such as IEEE, Springer and ACM. It is worth noting that Google Scholar gives full-text search against our keywords and validates that obtained research articles are relevant to our performed queries. It gives convenience by giving direct access to download research papers from relevant databases. To achieve significant coverage of research work related to UOWCs, we performed several queries on Google Scholar. First we search challenges in UOWCs, later we checked while combining research survey, review study, new trends, recent advances and limitations. We collected research articles and considered only those published 
in recent years in order to maintain good results and state-ofthe-art approach. Basically, we selected the recent research articles related to current challenges, new trends and issues in UOWCs. We made lists of relevant papers focusing same potential features and started reading abstracts and conclusions to achieve primary study related to this review. Our research methodology includes research questions, sources and keywords. A basic overview of our research procedure is displayed in Fig. 7.

\section{B. Research Questions}

We have composed a set of research questions (RQs) and motivation behind it. These questions will be helpful for researchers to recognize the research lack in this field. RQs and motivation is highlighted in Table VIII.

RQ1. Find out the theoretical properties of UOWCs in existing and future technologies and communication devices?

RQ2. Find out good simulations tools, best architecture design, suitable modulation technique, channel modeling scheme and proper communication model?

RQ3. Find out research investigations to mitigate effects which degrade the performance of UOWC system?

RQ4. What are the potential challenges and expected link damages and connectivity losses?

RQ4. How to recover delay in case of temporary communication loss?

RQ5. Compute a complete performance evaluation for different transmission ranges and water types to meet real world requirements?

RQ6. Test each solution against possible challenges in real world environment to achieve required performance of designed UOWC system?

\section{Search Strategy}

We have defined a good research strategy which will be helpful for researchers to retrieve specific research literature. Our research strategy includes research method, various search terms and different data resources. Our search strategy starts with fundamental steps to identify search terms and data resources:

1) Prefatory search to retrieve previous relevant literature.

2) Check research papers published in good journals and leading conferences.
3) Consult with researchers to find relevant data from conferences and journals.

4) Trial search based on prior defined research questions.

5) Using own learning and experience related to UOWCs.

\section{Search Method}

In our search strategies, we used two search methods: initial manual search and later automatic search. For manual search, we selected research papers which were published in specific venues as listed in Table IX.

For automatic data search, we used electronic data resources provided in Table $\mathrm{X}$ to obtain relevant papers.

\section{E. Search Terms}

We used search terms related to keywords, paper titles and abstracts found in differed electronic databases. Keywords are given in Table XI. We used below provided strategies to achieve the most relevant data by forming relevant search terms.

1) Find key terms according to research questions and study topic.

2) Make list of keywords mentioned in most relevant articles.

3) Search synonyms and major term.

4) Use Boolean operator "AND” in main terms.

5) Use Boolean operator "OR" in synonyms and corresponding terms.

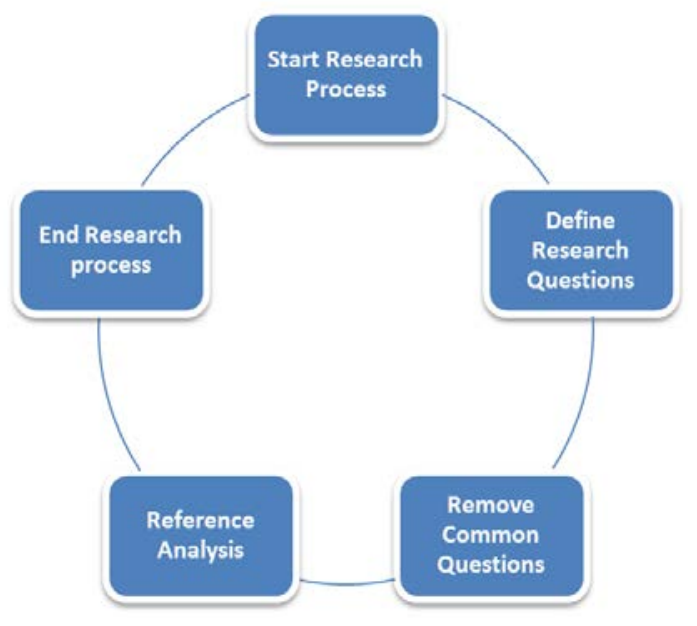

Fig. 7. Research Overview.

TABLE VIII. RESEARCH QUESTIONS

\begin{tabular}{|l|l|}
\hline Research Questions & Motivation \\
\hline RQ1 & It will help to find out current and future advancement in UOWC \\
\hline RQ2 & It will help to understand complete designing process of UOWCs \\
\hline RQ3 & It will help to investigate factors which degrade performance of UOWC system. \\
\hline RQ4 & It will help to recover communication and handle delay issue \\
\hline RQ5 & It will help to shape the model to meet required performance criteria. \\
\hline RQ6 & It will help to design system with real world applications. \\
\hline
\end{tabular}


TABLE IX. RESEARCH KEYWORDS

\begin{tabular}{|c|c|c|}
\hline Journal/ Conference & Venue & Acronym \\
\hline $\mathrm{J} 1$ & Chinese Optics Letter & COL \\
\hline $\mathrm{J} 2$ & Journal of Applied Physics & J APPL PHY \\
\hline J3 & Journal of Lightware Technology & J LIGHTWAVE TECHNOL \\
\hline $\mathrm{J} 4$ & Journal of Communication and Information Systems & JCIS \\
\hline J5 & IEEE Access & IEEE Access \\
\hline J6 & IEEE Communications Surveys and Tutorials & IEEE Commun. Surv \\
\hline $\mathrm{J} 7$ & IEEE Journal of Oceanic Engineering & IEEE J OCEANIC ENG \\
\hline J8 & Journal of Sensors & J. Sens. \\
\hline J9 & Photonics research & Photonics Res. \\
\hline $\mathrm{J} 10$ & Optics communications & Opt. Commun. \\
\hline J11 & IEEE Journal on Selected Areas in Communications & IEEE J SEL AREA COMM \\
\hline $\mathrm{J} 12$ & IEEE photonics journal & IEEE Photonics J. \\
\hline C1 & INTERNATIONAL CONFERENCE ON INFORMATION, COMMUNICATION AND NETWORK & ICICN \\
\hline C2 & International Conference on Transparent Optical Networks & ICTON \\
\hline C3 & International Conference on Optical Communications and Networks & ICOCN \\
\hline C4 & Australasian Symposium on Parallel and Distributed Computing & AusPDC \\
\hline C5 & International Conference on Advanced Infocomm Technology & ICAIT \\
\hline C6 & International Conference on Imaging, Signal Processing and Communication & ICISPC \\
\hline C7 & International Conference on Communications and Mobile Computing & ICCMC \\
\hline
\end{tabular}

TABLE X. ElECtronic DAtABASES

\begin{tabular}{|l|l|l|}
\hline Electronic Database & Search Items & Web Address \\
\hline Google Scholar & Keywords, Paper Title & https://scholar.google.com/ \\
\hline ScienceDirect & keywords,Paper title, abstract & https://www.sciencedirect.com/ \\
\hline IEEE eXplore & keywords,Paper title, abstract & www.ieeexplore.ieee.org \\
\hline ResearchGate & Keywords, Paper Title, Author & https://www.researchgate.net/ \\
\hline Sci-Hub & Keywords, Paper Title & https://sci-hub.tw/ \\
\hline ACM Digital Library & Keywords, Paper Title & http://portal.acm.org \\
\hline Elsevier & keywords,Paper title, abstract & https://www.elsevier.com/en-au \\
\hline ISI Web of Science & keywords,Paper title, abstract & http://www.webofknowledge.com \\
\hline SpringerLink & Keywords, Paper Title & http://www.springerlink.com \\
\hline
\end{tabular}

TABLE XI. RESEARCH KEYWORDS

\begin{tabular}{|l|l|}
\hline Keywords & Synonyms \\
\hline OWCs & Optical Wireless Communications \\
\hline UWC & Underwater Wireless Communication \\
\hline FSO & Free Space Optics \\
\hline UOC & Underwater Optical Communication \\
\hline AUV & Autonomous Underwater Vehicles \\
\hline UOWC & Underwater Optical Wireless Communication \\
\hline UOWC Link & Underwater Optical Wireless Communication Link, Link Budget, Link Configuration \\
\hline UOWC Challenges & Underwater Optical Wireless Communication Issues, Threats, Security Attacks \\
\hline UOWC Survey & Review, Overview and Study on UOWC \\
\hline
\end{tabular}


We defined inclusion and exclusion criteria to rectify this primary study to validate our research queries.

Inclusion Criteria:

- Any paper which declares its key findings related to challenges in UOWCs.

- Any paper which is published in 2010 or after 2010.

- Any paper which is written in English language only.

Exclusion Criteria:

- Article which do not meet specific required details against our approach.

- Articles which do not contain complete information about our desired research work.

- Articles which are extended by another article that we have selected already in our list. It gives us to find representative article towards our approach.

- Articles representing short paper, only abstract, an editorial, poster summary, panel summary or workshop summary. Such articles are missing with sufficient informative data.

- Articles such as white papers and technical reports as some research communities do not rectify such articles.

In short, our primary study must provide the right approach towards UOWCs with a strong focus on emerging trends, challenging issues, opportunities and limitations. After applying inclusion and exclusion criteria, we were able to find $\mathrm{N}=42$ research papers published in recent years. Different stages of our research process are given in Fig. 8.

\section{ANALYSIS STUDY}

We have collected analytical findings from several research publications on UOWCs. Researchers have used different analytical approaches and designed communication systems, channel modeling, coding and modulation techniques. Researchers are working on innovative services, technical aspects, real world applications and emerging trend of UOWCs. The research challenges are found in proper exploitation of underwater environment before communication and clear understanding of propagation characteristics of channel. Possible solutions are found in simulations, laboratory artificial aquatic environment or testbeds. There is need of an effective approach for system design, testing and analysis before its final deployment in wide ocean environment. The analytical study must give confidence and significant results that designed system will meet the required performance and efficiency. Researchers should consider these analysis questions.

1) Designed system will meet real world application requirements?

2) Which modulation technique and signal processing tools will provide high performance?

3) What is the required communication range for data transmission between source and destination?
4) What is the best path for communication without delay in information transmission?

5) How to overcome temporary connectivity losses while communication?

6) How to control power utility in order to enhance system reliability?

7) How to cope with any possible threats and security attacks to achieve secure communication in network?

8) Which factors will mainly affect the UOWCs design, communication architecture and deployment?

9) Which factors will mainly affect the UOWCs design, communication architecture and deployment?

10)How to tackle transceiver position and orientation in LOS and NLOS conditions?

The researchers should consider these questions and find our factors to develop analysis techniques and solutions. Their solutions must validate the real systems. Research community should carefully address the reliability and feasibility of optical links in underwater scenario.

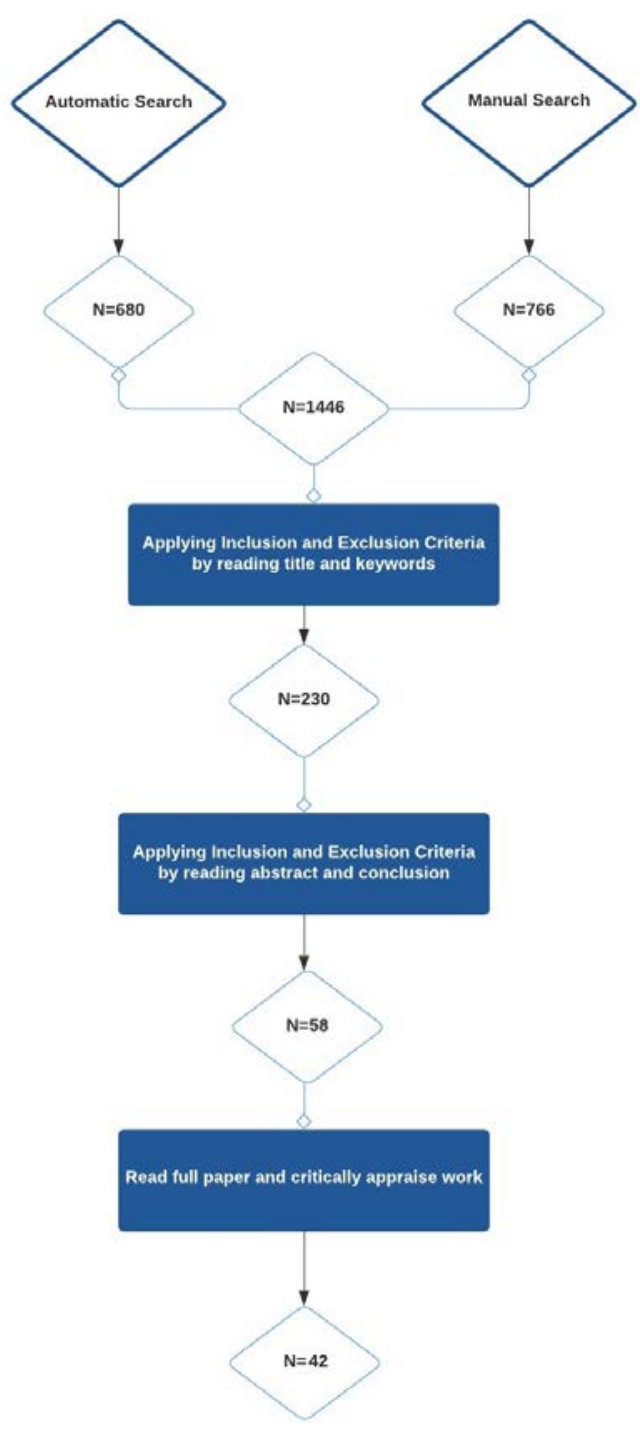

Fig. 8. Stages of Search Process. 


\section{RESEARCH VALIDITY AND LIMITATIONS}

We have found relevant research articles by using scientific keywords and references. We selected many papers from Google Scholar. In our exclusions, we selected relevant papers after reading title, abstract, conclusion and future aspects. A possibility of missing some recent papers or data still remains by using this keyword search, inclusion and exclusion methods. Here we have described the validity threats and limiting factors to this research study as follows:

1) Data sources: Our major sources for data collection were Google Scholar and ResearchGate. Though these sources are beneficial to search relevant data as they automatically fetch data from different databases, technical sources and academic publishers against single data query, but still we take it as a threat to validity as these sources suffer from certain limitations such as lack of search facility, partial control on content body and vulnerability to spam. To tackle this, we will include more sources, scientific search engines and publishers while extending our current work. Enabling this approach in extending our work, we are confident to get broader coverage of potential issues in UOWCs and will provide more statistical analysis.

2) Data collection process: While our data collection and analyzing process, we assigned a single researcher to read article and collect information from title, abstract and conclusion. It is a potential threat to validity of our findings and we tried to mitigate this concern through group discussion. We are aware that a single researcher can inject certain amount of inconsistencies in selected data. In future extended work, we will perform detailed statistical analysis through assigning this task to more than one researcher.

3) Selection of potential challenges: we tried to identify potential challenges of UOWCs. This investigation comes from preliminary analysis of different articles. Though we used an effective way to get appropriate analysis, still there can be more challenging factors. To alleviate this threat, one research member was assigned this task to keep a good record of possible challenges and issues discussed in each selected article. In the end, this approach provided confidence while validating our approach.

4) Time span: In inclusion criteria, we considered articles published after 2010 till now. Although it gives us right approach to find latest articles and key topics but it can affect the completeness of our search results as we did not include papers published before 2010. In future, we expect to involve more research recourses and considering articles published at a wide range of years.

\section{Optical Wireless Revolution: 5G AND 6G}

Scientists have been focusing on 6G communication after the successful development of $5 \mathrm{G}$ networks. $6 \mathrm{G}$ is expected to be launched between 2027 and 2030. Although 5G communication has many advantages such as high-quality video streaming, internet TV and faster communication [40]; however, it does not support ground-breaking technologies as it mainly focuses on performance. While privacy, secrecy and security are main features of 6G communication [41]. In addition, research community should focus on security and privacy issues which exploit wireless communication such as channel fading and noise. In underwater environment, 6G network will assist to deploy UWSN nodes in the form of AUVs and sensors connected with underwater base stations. Recently, some countries have made strategic plan on revolutionary advances of 6G. German and U.K. governments have invested in quantum technology. China has also made official announcement that Chinese researchers are focusing on the development of 6G. Researchers from USA have started working on terahertz-based 6G mobile networks. Terahertz frequency communication offers 1000 times higher capacity than $5 \mathrm{G}$ networks. One goal of $6 \mathrm{G}$ is to achieve ubiquitous connectivity by integrating satellite communication networks and underwater communications to provide global coverage.

RF-based technologies are insufficient to meet the demand of 5G/6G and IoT networks. Therefore, OWC technologies are the best complementary solution of RF networks. A very large optical band is a good alternative as it offers prominent features of high security, low latency, high data rate, high QoE, massive connectivity, low cost and low energy consumption. The coexistence of RF and optical wireless systems can achieve the goals of such networks. OWC technologies, such as FSO, OCC, LiFi and VLC can be effective to deploy future 5G/6G and IoT networks. We have summarized data rates and latency in current backhaul technologies in Table XII. Optical fiber communication provides the highest throughput among all existing technologies. However, a similar throughput can be achieved in FSO as it has similar type of transceivers. In future, FSO network will emerge as promising solution to support higher data rates in 5G/6G networks.

TABLE XII. DATA RATES AND LATENCIES

\begin{tabular}{|l|l|l|}
\hline Technology & Latency & Throughput \\
\hline Optical fiber & $<1 \mathrm{~ms}$ & $100 \mathrm{Gbps}$ \\
\hline FSO & $<1 \mathrm{~ms}$ & $40 \mathrm{Gbps}$ \\
\hline mmWave & $<1 \mathrm{~ms}$ & $10 \mathrm{Gbps}$ \\
\hline Microwave & $<1 \mathrm{~ms}$ & $1 \mathrm{Gbps}$ \\
\hline
\end{tabular}

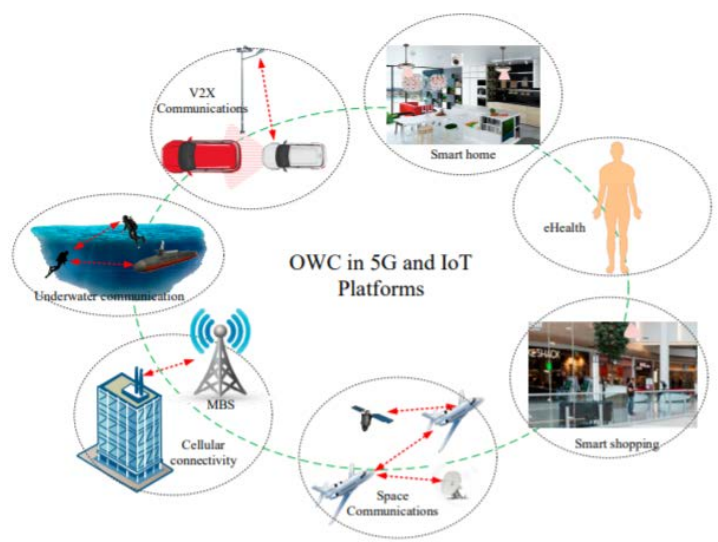

Fig. 9. OWC networks for the 5G/6G and IoT platforms [42]. 
OWC technologies can provide enormous number of connections through low-power LEDs to meet green aspects of 5G/6G and IoT networks. In addition, researchers should proficiently address some challenging issues such as atmospheric loss, flickering, inter-cell interference and frequent handover. A few important 5G/6G and IoT platforms using the OWC technologies are presented in Fig. 9.

\section{CONCLUSION}

There is a growing need to dig into elementary acuity to make UOWC a reality in future. For this, a detailed analysis of theoretical models is required. Herein, we have highlighted some key research areas with profound knowledge in our systematic review. We have carefully addressed UOWC strategies which influentially affect remote communication. However, research fraternity needs to focus to enhance data rate for video transmission at larger distances. UOWCs will offer potential features in real life applications and will put more impact in future. Our main objective was to identify key challenges for future research. It is concluded from our study that researchers are putting relevant efforts to handle challenges in system design, deployments, link configuration and analysis of UOWCs. For a reliable and efficient optical link, we have discussed system architecture, channel modeling, modulation techniques and operating wavelengths in this research study. We also conclude that rapid ongoing research in UOWC will be more conducive in better performance with game-changing features in future.

\section{FUTURE WORK}

We have highlighted a limitation of system with real time operating conditions and researchers must give specific concern as it will be beneficial to control remotely operated vehicles. As a precondition, research community should focus on developing intelligent modulation techniques to improve system performance. Future research should contemplate to involve Internet of Underwater Things (IoUT) to properly achieve real world applications. Researchers should pay attention to future 6G green communications. The coexistence of optical wireless and RF systems can meet the goals of such revolutions. Merging some latest underwater technologies with UOWC can bring promising results in future as shown in Fig. 10.

Besides all this, there is still room to develop cheap, adaptive, robust, highly stable, low powered and real time underwater optical sensors for an efficient UOWC system. In order to enhance overall robustness, adaptive techniques must be observed to save energy and optimize communication efficiency.

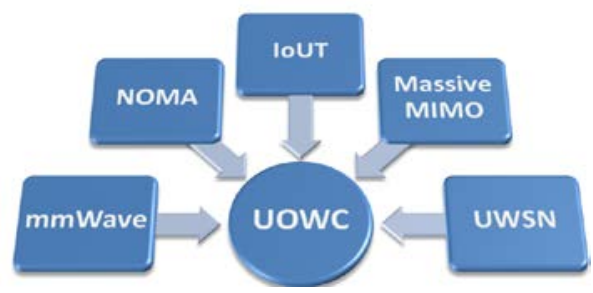

Fig. 10. Merging Underwater Technologies.

\section{REFERENCES}

[1] Cochenour, B.; Dunn, K.; Laux, A.; Mullen, L. Experimental measurements of the magnitude and phase response of high-frequency modulated light underwater. Appl. Opt. 2017, 56, 4019-4024.

[2] BlueComm Underwater Optical Communication. Available online: https://www.sonardyne.com/product/ bluecomm-underwater-opticalcommunication-system/ (accessed on 21 July 2020).

[3] Schirripa Spagnolo, Giuseppe, Lorenzo Cozzella, and Fabio Leccese. "Underwater Optical Wireless Communications: Overview." (2020): 2261.

[4] Al-Halafi, A.; Oubei, H.M.; Ooi, B.S.; Shihada, B. Real-Time Video Transmission Over Different Underwater Wireless Optical Channels Using a Directly Modulated 520nm Laser Diode. J. Opt. Commun. Netw. 2017, 9, 826-832.

[5] Han, S.; Noh, Y.; Lee, U.; Gerla, M. Optical-acoustic hybrid network toward real-time video streaming for mobile underwater sensors. Ad Hoc Netw. 2019, 83, 1-7.

[6] Sun, Xiaobin, et al. "A review on practical considerations and solutions in underwater wireless optical communication." Journal of Lightwave Technology 38.2 (2020): 421-431.

[7] Zeng, Zhaoquan, et al. "A survey of underwater optical wireless communications." IEEE communications surveys \& tutorials 19.1 (2016): 204-238.

[8] Kaushal, Hemani, and Georges Kaddoum. "Underwater optical wireless communication." IEEE access 4 (2016): 1518-1547.

[9] Ghassemlooy, Zabih, et al. "Emerging optical wireless communicationsadvances and challenges." IEEE journal on selected areas in communications 33.9 (2015): 1738-1749.

[10] Khalighi, Mohammad-Ali, et al. "Underwater wireless optical communication; recent advances and remaining challenges." 2014 16th International Conference on Transparent Optical Networks (ICTON). IEEE, 2014.

[11] Kaushal, Hemani, and Georges Kaddoum. "Free space optical communication: challenges and mitigation techniques." arXiv preprint arXiv:1506.04836 (2015).

[12] Zeng, Zhaoquan. A survey of underwater wireless optical communication. Diss. University of British Columbia, 2015.

[13] Liu, Weihao, Zhengyuan Xu, and Liuqing Yang. "SIMO detection schemes for underwater optical wireless communication under turbulence." Photonics Research 3.3 (2015): 48-53.

[14] Van Walree, Paul A. "Propagation and scattering effects in underwater acoustic communication channels." IEEE Journal of Oceanic Engineering 38.4 (2013): 614-631.

[15] Hou, Rui, et al. "A brief survey of optical wireless communication." dalam Proc. of the 13th Australasian Symp. on Parallel and Distributed Computing (AusPDC 2015), Januari. 2015.

[16] Lin, Aobo, et al. "Underwater wireless optical communication system using blue LEDs." Journal of Physics: Conference Series. Vol. 679. No. 1. IOP Publishing, 2016.

[17] Tian, Pengfei, et al. "High-speed underwater optical wireless communication using a blue GaN-based micro-LED." Optics express 25.2 (2017): 1193-1201.

[18] Almanee, Mohammad, and Suliman Aljutily. "A High-Efficiency Diverto-Diver Optical Communication System." 2019 3rd International Conference on Imaging, Signal Processing and Communication (ICISPC). IEEE, 2019.

[19] Peppas, Kostas P., Anthony C. Boucouvalas, and Zabih Ghassemloy. "Performance of underwater optical wireless communication with multipulse pulse-position modulation receivers and spatial diversity." IET Optoelectronics 11.5 (2017): 180-185.

[20] Rashed, Ahmed Nabih Zaki, and Hamdy A. Sharshar. "Performance evaluation of short range underwater optical wireless communications for different ocean water types." wireless personal communications 72.1 (2013): 693-708.

[21] Johnson, Laura J., Roger J. Green, and Mark S. Leeson. "Underwater optical wireless communications: depth dependent variations in attenuation." Applied optics 52.33 (2013): 7867-7873. 
[22] Johnson, Laura J., Roger J. Green, and Mark S. Leeson. "Underwater optical wireless communications: depth-dependent beam refraction." Applied optics 53.31 (2014): 7273-7277.

[23] Johnson, Laura J., et al. "Recent advances in underwater optical wireless communications." Underwater Technology 32.3 (2014): 167-175.

[24] Gkoura, Lydia K., et al. "Underwater optical wireless communication systems: A concise review." Turbulence Modelling Approaches-Current State, Development Prospects, Applications. InTech, 2017.

[25] Rosenkrantz, Etai, and Shlomi Arnon. "Optimum LED wavelength for underwater optical wireless communication at turbid water." Laser Communication and Propagation through the Atmosphere and Oceans III. Vol. 9224. International Society for Optics and Photonics, 2014.

[26] Alimi, Isiaka, et al. "Challenges and opportunities of optical wireless communication technologies." Optical communication technology 10 (2017).

[27] Keskin, Aysan, et al. "Effects of focused and collimated laser beams on the performance of underwater wireless optical communication links." 2015 4th International Workshop on Optical Wireless Communications (IWOW). IEEE, 2015.

[28] Anguita, Davide, et al. "Optical wireless underwater communication for AUV: Preliminary simulation and experimental results." OCEANS 2011 IEEE-Spain. IEEE, 2011.

[29] Boucouvalas, Anthony C., et al. "Underwater optical wireless communications with optical amplification and spatial diversity." IEEE Photonics technology letters 28.22 (2016): 2613-2616.

[30] Johnson, Laura J., Roger J. Green, and Mark S. Leeson. "The impact of link orientation in underwater optical wireless communication systems." 2014 Oceans-St. John's. IEEE, 2014.

[31] Hagem, Rabee M., et al. "Optical wireless communication for real time swimmers feedback: A review." 2012 International Symposium on Communications and Information Technologies (ISCIT). IEEE, 2012.
[32] Anguita, Davide, Davide Brizzolara, and Giancarlo Parodi. "Optical wireless communication for underwater wireless sensor networks: Hardware modules and circuits design and implementation." OCEANS 2010 MTS/IEEE SEATTLE. IEEE, 2010.

[33] Vali, Zahra, et al. "Experimental study of the turbulence effect on underwater optical wireless communications." Applied optics 57.28 (2018): 8314-8319.

[34] Giuliano, Giovanni. Underwater optical communication systems. Diss. University of Glasgow, 2019.

[35] S. Arnon, "Underwater optical wireless communication network,” Opt. Eng., vol. 49, Jan. 2010, Art. no. 015001.

[36] X. Yi, Z. Li, and Z. Liu, "Underwater optical communication performance for laser beam propagation through weak oceanic turbulence,” Appl. Opt., vol. 54, no. 6, pp. 1273-1278, 2015.

[37] Y. Dong, S. Tang, and X. Zhang, "Effect of random sea surface on downlink underwater wireless optical communications,” IEEE Commun. Lett., vol. 17, no. 11, pp. 2164-2167, Nov. 2013.

[38] Hassnain, S. A., M. J. Mughal, and Q. A. Naqvi. "Layered Chiral Spheres with Zero Backscattering." 2019 Photonics \& Electromagnetics Research Symposium-Fall (PIERS-Fall). IEEE, 2019.

[39] Hassnain, S. A., M. J. Mughal, and Q. A. Naqvi. "Analysis of Effective Medium Parameters on Polarizability of Homogeneous Chiral Sphere." 2019 Photonics \& Electromagnetics Research Symposium-Fall (PIERSFall). IEEE, 2019.

[40] Information can be accessed at: https://www.eurekalert.org/ pub_releases/2020-02/kauo-pia020720.php

[41] Dang, Shuping, et al. "What should 6G be?." Nature Electronics 3.1 (2020): 20-29.

[42] Chowdhury, Mostafa Zaman, et al. "The Role of Optical Wireless Communication Technologies in 5G/6G and IoT Solutions: Prospects, Directions, and Challenges."Applied Sciences 9.20 (2019): 4367. 